\title{
TRANSLATION, ADAPTATION AND VALIDATION OF THE BULGARIAN VERSION OF THE SIDAED QUESTIONNAIRE
}

\author{
Rumyana N. Kuzmanova, \\ Irina Z. Stefanova, \\ Irena V. Velcheva, \\ Katerina I. Stambolieva ${ }^{2}$
}

Department of Epilepsy, University Hospital of Neurology and Psychiatry "St. Naum", Sofia

${ }^{1}$ Department of Cognitive Psychophysiology, Institute of Neurobiology, Bulgarian Academy of Science Sofia

\author{
Corresponding author: \\ Rumyana Kuzmanova \\ Department of Epilepsy, \\ University Hospital of Neurology and \\ Psychiatry "St. Naum", \\ 1, Louben Roussev str. \\ Sofia, 1113 \\ Bulgaria \\ e-mail: katerina_stambolieva@yahoo.com
}

Received: February 25, 2015

Revision received: March 18, 2015

Accepted: May 18, 2015

\section{Summary}

The aim of the study is the translation, adaptation and validation of Side effects of antiepileptic drugs questionnaire in Bulgarian language (SIDAED-BG) in order to use it for objective monitoring of patients with epilepsy. One hundred and thirty one patients (mean age $40.13 \pm 13.37$ years) took part in the investigation. The internal consistency and test-retest reliability were tested by Cronbach's $\alpha$ and ICC estimations. The convergent construct validity was evaluated by estimating the correlation of SIDAED-BG with the QOLIE-89 and the discriminant validity - by evaluation of the difference between SIDAED-BG scores and clinical parameters such as type of epilepsy using Kruskal-Wallis ANOVA. The Cronbach's $\alpha$ of the total scale was 0.93 . The testretest reliability was higher and determined the strong positive correlations between the first and second examination. The SIDAED-BG questionnaire showed good internal consistency (Cronbach's alpha ranged from 0.37 to 0.86 ) and the scores significantly correlated with other questionnaires such as QOLIE-89 and showed a good discriminative validity between groups with different levels of self-assessed adverse effects of antiepileptic drugs. The Bulgarian version of SIDAED is a reliable and valid tool in assessing the patient-reported adverse effects of antiepileptic drugs and their impact on the patient's outcome.

Key words: SIDAED questionnaire, epilepsy, adverse events, antiepileptic treatment, quality of life in epilepsy inventory

\section{Introduction}

The goals of the long-term antiepileptic treatment are to gain the best control of seizures with the least adverse drug events. Many studies have shown that quality of life (QoL) and global health ratings are related to patient reported adverse effects (AE) and tolerability of antiepileptic drugs (AED) [1-9]. The Side Effects of Antiepileptic Drugs Questionnaire (SIDAED) is one of the recommended instruments for assessment of patient-reported adverse effects of AED in adults with epilepsy [6]. Reliability of the SIDAED was not reported.

There are no validated instruments or studies in Bulgarian emphasizing the reliability and validity of 
questionnaires measuring AE of AED in adults with epilepsy.

The aim of the present study is the translation, adaptation and validation of SIDAED questionnaire in Bulgarian in order to use it as a reliable instrument for routine and objective monitoring of patients with epilepsy.

\section{Patients and Methods}

The study group consisted of 131 patients from the Epilepsy Department of the University Hospital "St. Naum", Sofia, Bulgaria. Selection of patients was based on several criteria: (1) Diagnosis of epilepsy according to the ILAE criteria for more than 1 year; (2) Age of more than 18 years; (3) Lack of cognitive impairment tested with Mini-Mental State Examination Score $\geqslant 28$ when used as a screening scale; (4) Stable doses of AEDs for at least three months prior to study entry. Patients with another progressive neurological or psychiatric disease or some other chronic severe physical comorbidity (diabetes, asthma, heart, renal or hepatic failure etc.) on stable concomitant medication were excluded from the study.

All subjects gave a signed informed consent to participate in the study, which was approved by the local Ethic Committee in accordance with the ethical standards of the Helsinki declaration.

After obtaining agreement with Prof. Uijl and Prof. van Donselaar, the English version of the SIDAED questionnaire was translated by two independent medical experts with good knowledge of English and experience in adaptation of clinical tests, and re-translated in English by two independent translators who were unaware of the original version. No major differences between the two translations were found.

The preliminary Bulgarian version of SIDAED (SIDAED-BG) was edited according to the rules of the Bulgarian language for semantic and grammatical accuracy. In order to identify problems related to comprehension, the SIDAED-BG was administered to a pilot sample of ten patients with epilepsy. After performing and evaluating the pilot study, we came to the conclusion that two of the items should be modified so that the inventory would better suit our purpose. First, we observed that the word "light-headed" had to be changed to "dizzy." Second, "making love" was replaced by "sex". All questions were well accepted.
The original SIDAED questionnaire consists of 46 items with possible AED-related complaints distributed into ten domains: General Central Nervous System (CNS), Behavior (increased irritability), Depressive Symptoms, Cognitive Function, Motor Problems and Coordination, Visual Complaints, Headache, Cosmetic and Dermatological Complaints, Gastrointestinal complaints and Sexuality and Menses [10, 11]. While creating SIDAED-BG, we found that item 45 "I often suffer from stomach problems" refers to the category of the gastrointestinal complaints, and not to the cosmetic and dermatological complaints. That was the reason to include this item in "Gastrointestinal complaints" domain of the SIDAED-BG questionnaire (Table 1). The gender-specific item ("menses") was excluded from the domain "Sexuality and Menses". This domain in SIDAED-BG questionnaire contains two unisex questions and was named "Sexuality" (Table 1).

The severity of each complaint is rated on a four-point Likert scale (no problem, mild, moderate, or serious problem), as well as in the original SIDAED questionnaire. A score ranging from 0 to 135 may be calculated to measure the total adverse event burden.

All the patients were asked to fill in two questionnaires: SIDEAD-BG (Table 1) and the Bulgarian version of the QOLIE-89 that consists of 89 questions divided into seventeen domains [12] (Table 2).

\section{Statistical analyses}

Descriptive statistics were used to calculate the mean scores of the SIDAED-BG scale and demographic data. The score distribution of each item was evaluated for investigating the possible ceiling and floor effects. The internal consistency of SIDAED-BG scale was determined by estimating Cronbach's $\alpha$ coefficient and itemtotal correlation (Spearman's rank correlation $<0.25$ were considered to be weak, values of 0.76 and higher were considered to indicate a strong relationship). In general, Cronbach's $\alpha>0.7$ (Nunnally's criterion) indicates high levels of internal consistency $[13,14]$.

The test-retest reliability was evaluated by calculating the ICCs (value of 0.0 indicated 'no reliability', $>0.75$ was defined as 'good' and 1.0 as 'perfect').

The convergent construct validity was tested with estimation of the correlation of the 
Table 1 SIDAED list of subjective complaints according to Prof. Uijl and Prof. van Donselaar $[10,11]$

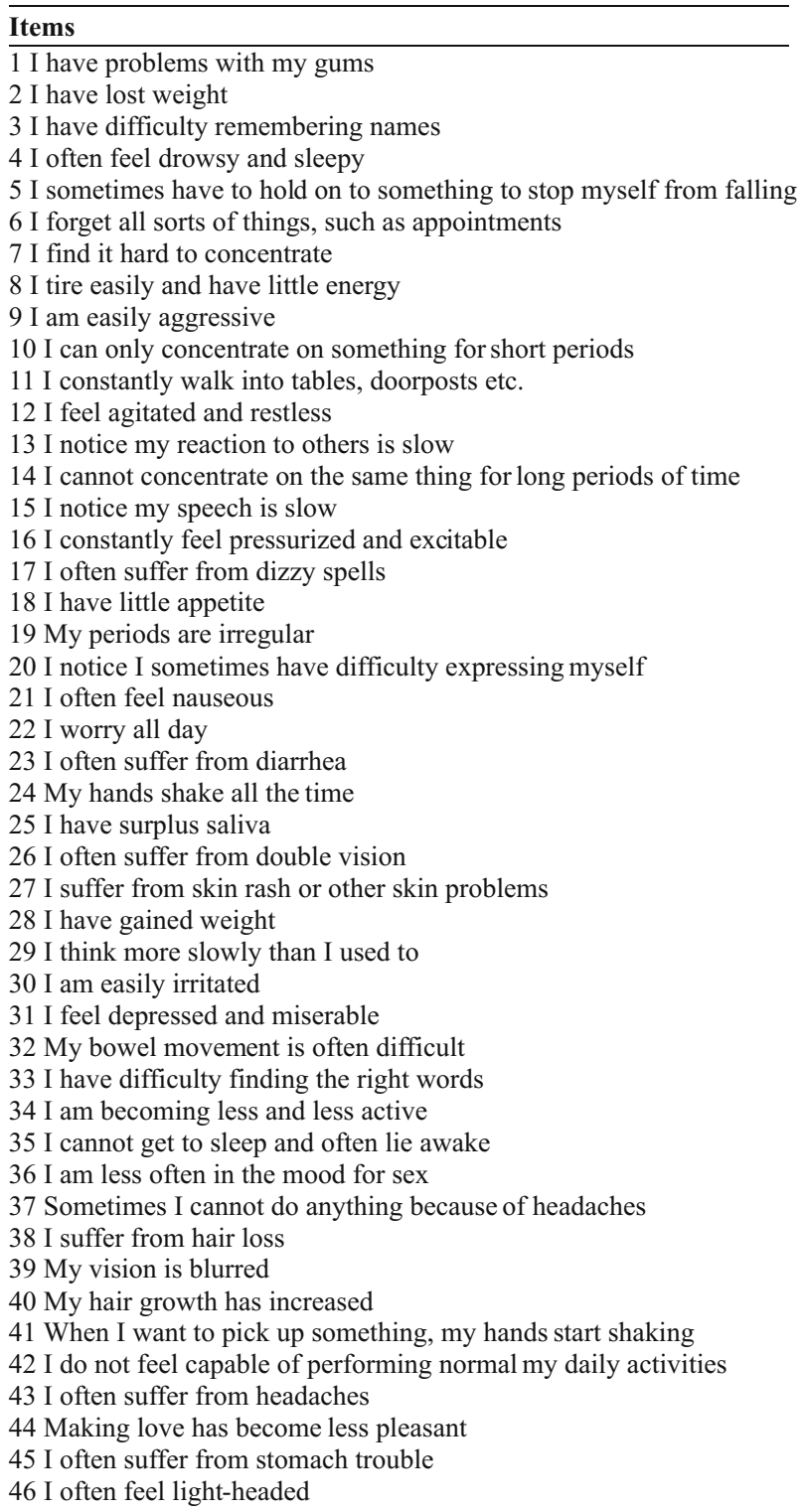

Subscales of SIDAED-BG

General CNS $(4,8,14,42$, items),

Behavior (increased irritability) $(9,12,16,30)$,

Depressive Symptoms $(22,31,34,35)$,

Cognitive Function (3, 6, 7, 10, 13, 15, 20, 29, 33),

Motor Problems and Coordination $(5,11,17,24,41,46)$,

Visual complaints $(26,39)$,

Headache $(37,43)$

Cosmetic and Dermatological Complaints $(1,25,27,38,40)$,

Gastrointestinal Complaints $(2,18,21,23,28,32,45)$

SIDAED-BG with QOLIE-89. The discriminant validity was established by evaluation of a difference between the SIDAED -BG scores and a clinical parameter, such as type of epilepsy, using Kruskal-Wallis ANOVA.

The analyses were made using the computer software Statistica 8.0 for Windows (Stat Soft Inc.USA).

\section{Results}

The sample of 131 adults comprised of 57 males and 74 females (mean age of $40.13 \pm 13.37$ years) and mean duration of epilepsy $15.61 \pm 9.45$ years).

All patients completed the questionnaire by hand. There was no missing data in the completed questionnaire, with the exception of the questions related to sexuality which were not completed by $47 \%$ of the patients. This is the reason for excluding the domain "sexuality" from the analysis of SIDAED-BG.

The main parameters and the parameters reflecting the internal consistency and test-retest reliability of the questions in the subscales of the SIDAED are shown in Table 3.

The scores for all domains were not normally distributed (Table 3). The internal consistency of the total score was 0.93 . The Cronbach's $\alpha$ coefficients ranged from 0.37 to 0.86 (lowest level of the "Cosmetic and dermatological complaints" and highest of the "Cognitive function" sub-scales).

The Intraclass Correlations (ICCs) were applied to evaluate the test-retest reliability and showed no significant differences between the scores. The ICCs varied between 0.77 (for "Cosmetic and dermatological complaints") and 0.91 (for "Cognitive function") and were higher than the recommended value of 0.75 [14]. That showed strong positive correlations between the first and the second examination, which referred to the reliability of the scale (Table 3 ).

The correlation between the total score of the SIDAED-BG and the "Overall Health" scale of the QOLIE-89 was - 0.596. The "General CNS" scale showed highest correlation with all scales of the QOLIE-89, except for the "Social support" scale (Table 2).

The Kruskal-Wallis ANOVA with the three levels (idiopathic, cryptogenic and symptomatic type of epilepsy) showed a significant effect of the self-reported complaints on the SIDAED-BG total score $(\mathrm{H}(2,131)=8.89, \mathrm{p}<0.05)$ and the subscales: General CNS (H $(2,131)=13.4$, $\mathrm{p}<0.05)$; Depressive symptoms $(\mathrm{H}(2,131)=$ $6.58 ; \mathrm{p}<0.05)$; Cognitive function $(\mathrm{H}(2,131)$ $=6.67, \mathrm{p}<0.05) ;$ Motor problems and 


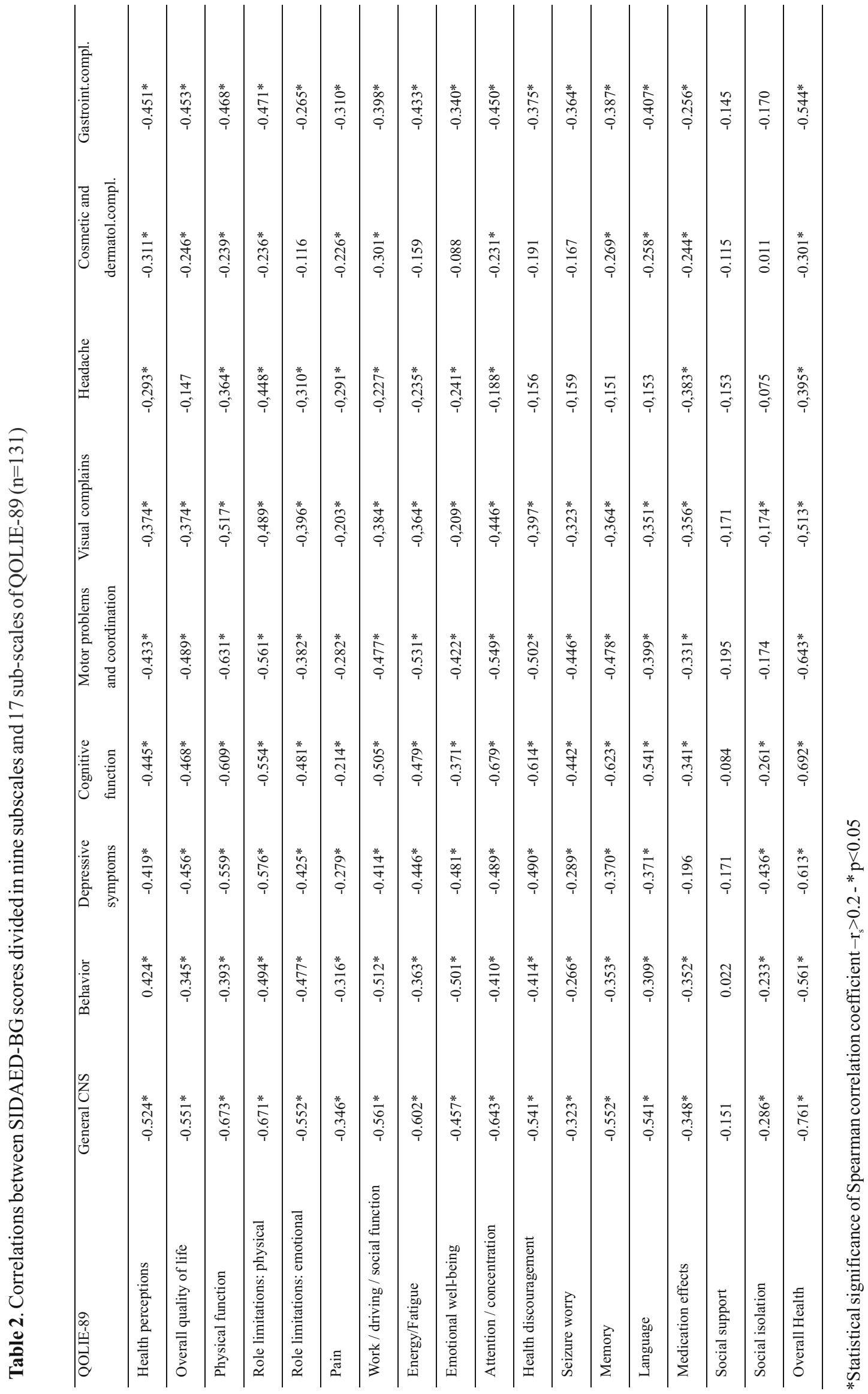




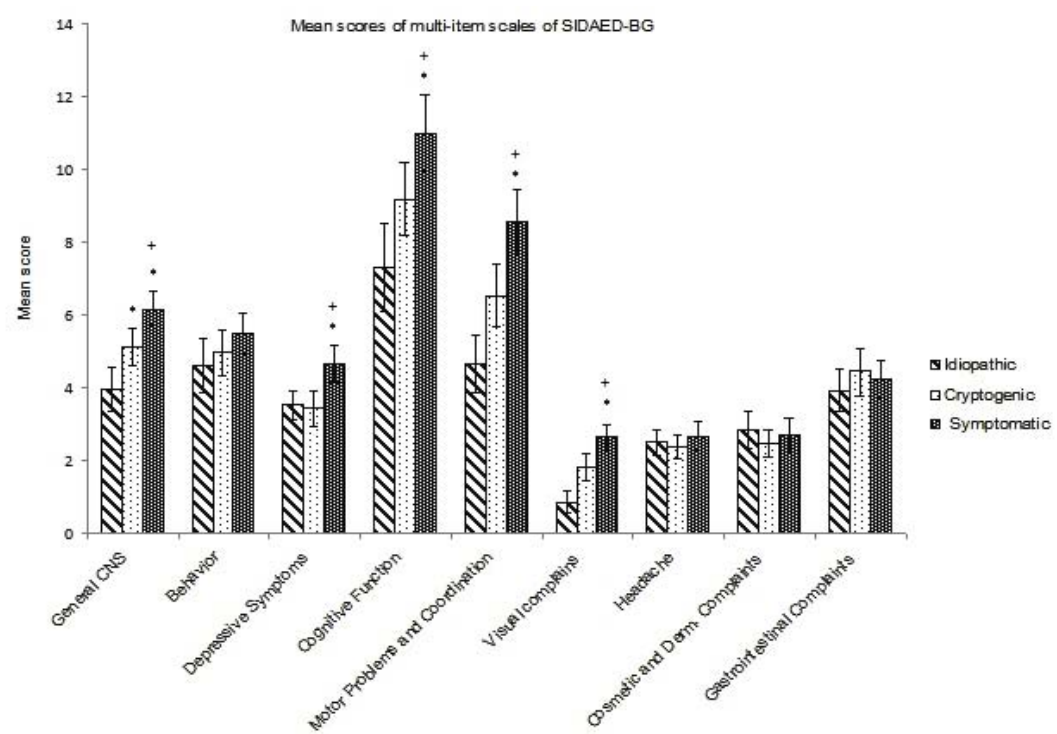

Figure 1. Mean scores of the multi-item subscales of the SIDAED-BG for subjective complains of the Bulgarian patients with epilepsy. The data is presented by means \pm SEM. The significant differences between the group with idiopathic epilepsy and other groups $(\mathrm{p}<0.05)$ are noted with $*$, while the differences between the groups with symptomatic and cryptogenic epilepsy $(\mathrm{p}<0.05)$ are noted with + .

coordination $(\mathrm{H}(2.131)=14.07, \mathrm{p}<0.01)$. A post hoc Mann-Whitney U-test showed a significant difference between the total SIDAED-BG score and the four sub-scales: General CNS, Depressive symptoms, Cognitive function and Motor problems and Co-ordination scores of patients with symptomatic epilepsy and the groups with idiopathic and cryptogenic epilepsy (Figure1). The results suggest that the SIDAED-BG questionnaire shows a good discriminative validity between the groups with different types of epilepsy.

\section{Discussion}

This is the first validated version of the SIDAED questionnaire.

The sub-scale "Cosmetic and dermatological complaints" shows low reliability and low convergent and discriminate construct validity in Bulgarian patients with epilepsy. This defines this sub-scale of the SIDAED-BG questionnaire as not sufficiently informative and reliable. The most informative for the evaluation of the subjective complaints in the Bulgarian population were the sub-scales: Cognitive function, Behavior (increased irritability), General CNS, Motor problems and Coordination, and Depressive symptoms. The prevalence of cognitive and general CNS complaints in our study group is in accordance with earlier studies $[1,10,15]$.
The high correlation between the total score of the SIDAED-BG and the "Overall Health" scale of the QOLIE-89 demonstrates the relationship between the unwanted drug effects and the whole QoL, confirmed by other authors as well $[1,2,5$, $8,12]$.

Higher scores of the scale and sub-scales for the group with symptomatic epilepsy present the more severe course of the illness and drug burden in this population of patients.

There are several limitations of the current study. The main limitation of our work is that the patient sample was selected from epilepsy clinics at a University hospital and these subjects might not be representative epilepsy patients for the general population. Additional study limitations include the lack of an independent control group, as well as aspects of personality that were not investigated.

\section{Conclusion}

The results of this study confirm the reliability of the SIDAED-BG questionnaire with a new construction (43 items, divided into nine domains) and provide a solid rationale for recommending the use of the Bulgarian version for assessing the self-reported AE of AED, and their influence on the patients' outcome. 
J Biomed Clin Res Volume 8 Number 1, 2015

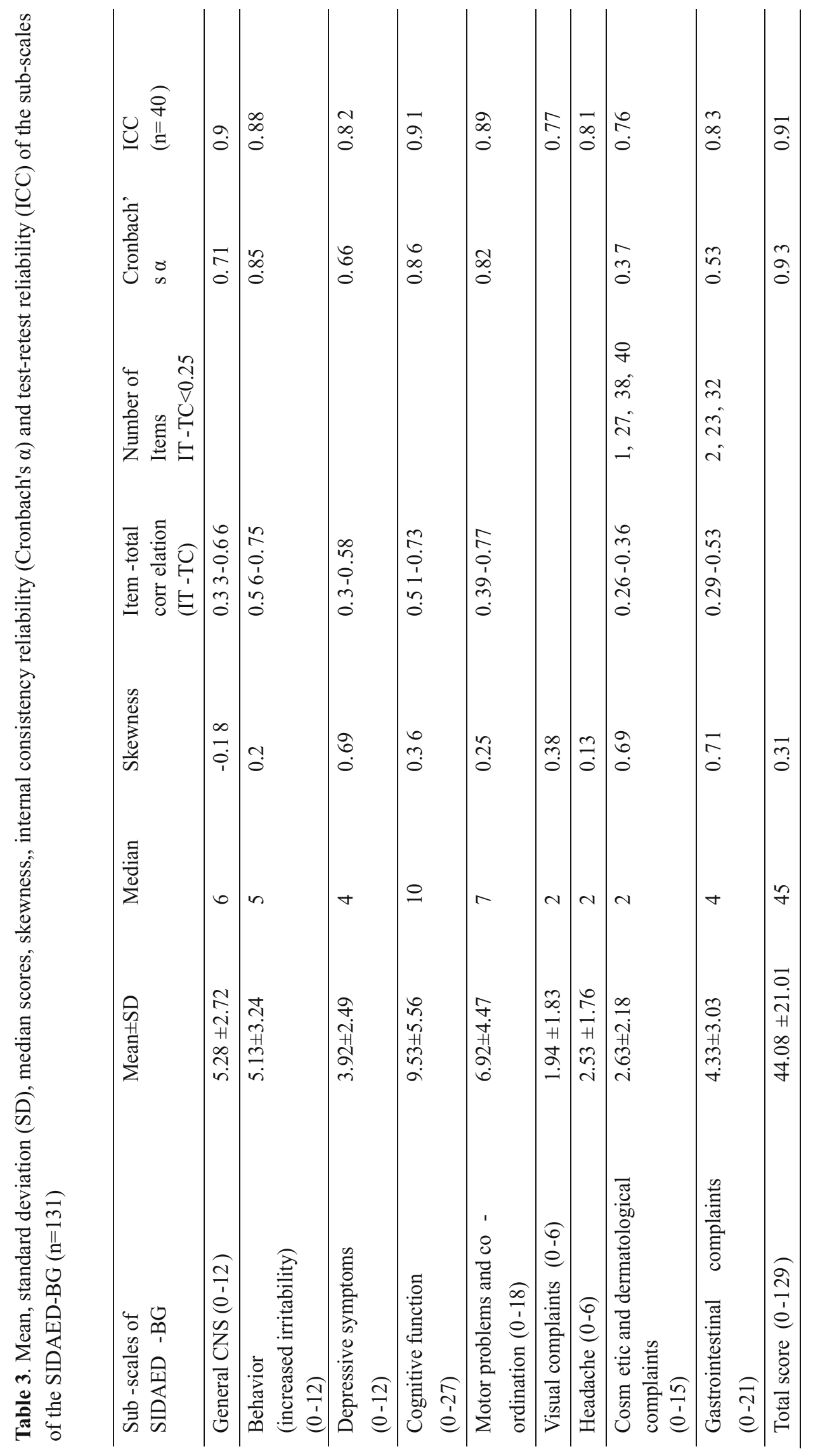




\section{References}

1. Baker GA, Jacoby A, Buck D, Stalgis C, Monet D. Quality of life of people with epilepsy: a European study. Epilepsia. 1997;38(3):353-62.

2. Cramer JA, Brandenburg NA, Xu X, Vera-Llonch M, Oster G. The impact of seizures and adverse effects on global health ratings. Epilepsy Behav. 2007;11(2):179-84.

3. Fisher RS, Vickrey BG, Gibson P, Hermann B, Penovich P, Scherer A et al. The impact of epilepsy from the patient's perspective II: views about therapy and health care. Epilepsy Res. 2000;41(1):53-61.

4. Gilliam F. Optimizing health outcomes in active epilepsy. Neurology. 2002;58(Suppl. 5):9-19.

5. Luoni C, Bisulli F, Canevini MP, De Sarro G, Fattore C, Galimberti CA, et al. Determinants of health-related quality of life in pharmacoresistant epilepsy: results from a large multicenter study of consequtively enrolled patients using validated quantitative assessments. Epilepsia. 2011;52(12):2181-91.

6. May TW, Brandt C, Kassel J. Evaluation of a selfreport questionnaire for the assessment of adverse effects of antiepileptic drugs. Epilepsia. 2009;50 (Suppl 4):104.

7. Perucca P, Carter J, Vahle V, Gilliam FG. Adverse antiepileptic drug effects: toward a clinically and neurobiologically relevant taxonomy. Neurology. 2009;72(14):1223-9.

8. Taylor RS, Sander JW, Taylor RJ, Baker GA. Predictors of health related quality of life and costs in adults with epilepsy: a systematic review. Epilepsia. 2011;52(12):2168-80.
9. Nixon A, Kerr C, Breheny K, Wild D. Patient Reported Outcome (PRO) assessment in epilepsy: a review of epilepsy-specific PROs according to the Food and Drug Administration (FDA) regulatory requirements. Health Qual Life Outcomes. 2013;11:38.

10. Uij1 SG, Uiterwaal CS, Aldenkamp AP, Carpay JA, Doelman JC, Keizer K, et al. A cross-sectional study of subjective complaints in patients with epilepsy who seem to be well-controlled with antiepileptic drugs. Seizure. 2006;15(4): 242-248.

11. Uijl SG, Uiterwaal CS., Aldenkamp AP, Carpay JA, Doelman JC, Keizer K, et al. Adjustment of treatment increases quality of life in patients with epilepsy: a randomized controlled pragmatic trial. Eur J Neurol. 2009;16(11):1173-7.

12. Viteva EI, Zachariev ZI, Semerdzhieva MA. Validation of the Bulgarian version of the quality of life in epilepsy inventory (QOLIE-89). Folia Med. 2010;52(1):34-9.

13. Cronbach LJ. Coefficient alpha and the internal structure of tests. Psychometrika. 1951;16(3):297334.

14. Nunnally JC, Bernstein IH. Psychometric theory. 3th ed. McGraw-Hill; 1994.

15. Carpay JA, Aldenkamp AP, van Donselaar CA. Complaints associated with the use of antiepileptic drugs: results from a community-based study. Seizure. 2005;14:198-206. 